\title{
Simultaneous Determination of Combined and Free Concentrations of Atorvastatin and its Major Metabolite in Vitro and in Vivo Based on Ultrafiltration Coupled with UPLC-MS/MS Method: Application to Protein Binding Rates and Metabolism Ability Study in Uremic Hemodialysis Patients.
}

Ming-Chen Cao

The Affiliated Hospital of Qingdao University

Meng-Xiang Fang

Huangdao District Second Hospital of Traditional Chinese Medicine

Li Wang

Shandong University

Xin Huang ( 13791120711@126.com )

Shandong University

Bo-Hao Tang

Shandong University

Hai-Yan Shi

Shandong University

Yi Zheng

Shandong University

Wei Zhao

Shandong University

Research Article

Keywords: Atorvastatin, UPLC-MS/MS, Uremia, metabolite, protein binding rate

Posted Date: October 26th, 2021

DOl: https://doi.org/10.21203/rs.3.rs-951801/v1 
License: (c) (i) This work is licensed under a Creative Commons Attribution 4.0 International License. Read Full License 


\section{Abstract}

Objectives: A rapid, accurate and specific ultrafiltration with ultra performance liquid chromatographic tandem mass spectrometry method has been validated for the simultaneous determination of the protein binding rate of atorvastatin for uremia patients.

Methods: The plasma samples were centrifuged at $6000 \mathrm{r} \cdot \mathrm{min}-1 \mathrm{for} 15 \mathrm{~min}$ at $37^{\circ} \mathrm{C}$ and the ultrafiltrate was collected. An ACQUITY UPLC ${ }^{\circledR}$ BEH C18 Column with gradient elution of water ( $0.1 \%$ formic acid) and acetonitrile was used for separation at a flow rate of $0.4 \mathrm{~mL} / \mathrm{min}$. The calibration curves of two analytes in serum showed excellent linearity over the concentration ranges of $0.05-20.00 \mathrm{ng} / \mathrm{mL}$ for atorvastatin, and $0.05-20.00 \mathrm{ng} / \mathrm{mL}$ for orth-hydroxy atorvastatin, respectively.

Results: This method was validated according to standard FDA and EMA guidelines in terms of selectivity, linearity, detection limits, matrix effects, accuracy, precision, recovery, and stability ${ }^{[1-2]}$. This assay can be easily implemented in clinical practice to determine free and combined concentration of atorvastatin in uremic plasma. The final result showed that the average protein binding rate of plasma in uremic patients plasma was $(86.58 \pm 2.04) \%$, RSD $(\%)=1.98$, while the protein binding rate in plasma of patients with normal renal function was $(97.62 \pm 1.96) \%$, RSD $(\%)=2.04$, there was a significant difference in the protein binding rate of different types of plasma $(P<0.05)$, and the protein binding rate decreases with increasing creatinine until it is stable at nearly $80 \%$. The mean metabolite/prototype ratio of atorvastatin for patients with normal renal function and uremia were 1.085 and 0.974 , respectively, suggesting that the metabolic process of atorvastatin in uremic patients may be inhibited.

Conclusions: In this study, the total concentration of atorvastatin in uremic patients did not change significantly, but due to the decrease of protein binding rate, the concentration of free drug may fluctuate drastically, and the increase of free drug concentration will increase the drug distribution in the liver or muscle tissue. Uremic patients who was taking atorvastatin should be monitored regularly, ensure the safety of medication for this particular group.

\section{Introduction}

Atorvastatin was a potent synthetic inhibitor of 3-hydroxy-3-methylglutaryl (HMG)-CoA reductase, the ratelimiting enzyme in cholesterol biosynthesis, had been widely used in many countries for the treatment of

hyperlipidemia because of its high efficacy and safety ${ }^{[3]}$. Recent studies had found that statins played an important role in the prevention and treatment of kidney disease, and had certain renal protective effects $^{[4]}$, thence occupied a large proportion of hypolipidemic drugs used in uremia hemodialy patients. Atorvastatin is extensively bound to plasma proteins (95 98\%), and conventional hemodialysis cannot be effectively eliminated. There may be a risk of drug accumulation in uremia patients, and the protein binding rate of the drug may change, increasing the risk of medication. The incidence of rhabdomyolysis in severe adverse reactions of atorvastatin has been reported to increase with increasing blood drug concentration $^{[5]}$. The Lipitor ${ }^{\circledR}$ (Atorvastatin Calcium) specification states that kidney disease does not 
have any effect on atorvastatin plasma concentrations and lipid-lowering effects. Renal dysfunction does not require adjustment of dose, but uremic dialysis patients need the dose adjustment is not yet clear.

In this study, a rapidly and precisely ultrafiltration and UPLC-MS/MS method will be established, and was used to study the changes in the protein binding rate of atorvastatin in uremia hemodialysis patients in vitro. Symptoms of dialysis patients safe and effective application of the drug to provide a reference basis to protect this special group of drug safety.

\section{Experimental}

\section{CHEMICALS AND MATERIALS}

The standards of atorvastatin (Lot number 132320-201405) were purchased from the National Institutes for Food and Drug Control (Beijing, China).The standards of orth-hydroxy atorvastatin(Lot number 241420 ) were purchased from the TLC PharmaChem., Inc. (Canada). The reference standard of chlorzoxazone (internal standard, IS; Lot number 133874-201508) was purchased from the National Institutes for Food and Drug Control (Beijing, China). HPLC-grade methanol, acetonitrile and methyl tertbutyl ether (MTBE) were obtained from Sigma-Aldrich (St. Louis, USA). Formic acid and ammonium acetate(Analytical reagent) was obtained from Beijing Chemical Factory (Beijing, China). The water used in the Laboratory was Ultra-pure water. All other chemicals used were of analytical reagent grade.

Amicon Centrifree $®$ UFC501096 micropartition devices with a filter membrane of 10,000KDa molecular weight cut-off were purchased from Millipore (Bedford, MA, USA).

Plasma was obtained from drug-free volunteers with normal renal function and uremia hemodialysis patients.

\section{PREPARATION OF STANDARD SOLUTIONS}

Stock solutions of atorvastatin $(1 \mathrm{mg} / \mathrm{mL})$ ]orth-hydroxy atorvastatin $(1 \mathrm{mg} / \mathrm{mL})$ and chlorzoxazone as internal standard $(100 \mu \mathrm{g} / \mathrm{mL})$ were dissolved in methanol. The stock solutions were protected from light and kept at $4^{\circ} \mathrm{C}$ until used. The stock solutions were successively diluted with methanol:water (50:50, $\mathrm{V}: \mathrm{V})$ to prepare working solutions just prior to use. These solutions were spiked into drug-free human plasma samples to give final concentrations of $0.050,0.10,0.20,0.50,1.00,2.50,5.00$ and $20.00 \mathrm{ng} / \mathrm{mL}$.

\section{PREPARATION OF PLASMA SAMPLES}

Each plasma sample $(400 \mu \mathrm{L})$ was immediately mixed with $20 \mu \mathrm{L}$ of methanol-water $(50: 50, \mathrm{v} / \mathrm{v}), 40 \mu \mathrm{L}$ of the internal standard solution and $10 \mu \mathrm{L}$ of $0.1 \mathrm{M}$ sodium acetate solution, vortex for $30 \mathrm{~s}$, then mixed with MTBE $1 \mathrm{~mL}$. After vortex mixing for $1 \mathrm{~min}$ and centrifugation at $4^{\circ} \mathrm{C}$ and $6000 \mathrm{r}$.min- 1 for $10 \mathrm{~min}, 800$ $\mu \mathrm{L}$ of the upper MTBE fraction was transferred into a $1.5 \mathrm{~mL}$ EP tube. The upper MTBE fraction was evaporated to dryness under a gentle stream of nitrogen. The residue was redissolved in $100 \mu \mathrm{L}$ of 
methanol-water (50:50, v/v), vortexed for $1 \mathrm{~min}$, then centrifuged at $13000 \mathrm{r} \cdot \mathrm{min}-1$ for $10 \mathrm{~min}$, and the supernatant was sampled, and $10 \mu \mathrm{L}$ was injected into the UPLC system for analysis.

\section{Instrumentation and chromatographic conditions}

Analysis were performed on an MS/MS system consisting of AB SCIEX QTRAP 6500plus MS/MS, and the UPLC system consisted of a binary pump, an autosampler and an online degasser were used for UPLC-MS/MS analysis (SCIEX, MA, USA). Separation was achieved using an ACQUITY UPLC® BEH C18 Column (1.7um, 2.1*30mm, I.D) and ACQUITY UPLC ${ }^{\circledR}$ BEH C18 VanGuardTM Pre-Column 3/PK (1.7um, $2.1 \star 5 \mathrm{~mm}$ ) purchased from Waters (Massachusetts, American). Gradient elution was performed using water $(0.1 \%$ formic acid, $\mathrm{PH} 2)(\mathrm{A})$ and acetonitrile (B) at a flow-rate of $0.4 \mathrm{~mL} / \mathrm{min}$. The mobile phase was filtered prior to use through a $0.22 \mu \mathrm{m}$ Millipore filter paper (Billerica, USA) and degassed ultrasonically (Branson- Emerson USA) for $10 \mathrm{~min}$. The gradient program was as followed: $0.0-0.5 \mathrm{~min} 90 \% \mathrm{~A}$; 0.5 min-1.5 min, $90 \%$ A to $5 \% A ; 1.5-2.0 \mathrm{~min}, 5 \% \mathrm{~A} ; 2.0-2.1 \mathrm{~min}, 5 \% \mathrm{~A}$ to $90 \% \mathrm{~A} ; 2.1 \mathrm{~min}-3.5 \mathrm{~min}, 90 \% \mathrm{~A}$. The pressure of the UPLC apparatus was in the range of 9.5-19.5 MPa.

The ESI was operated in positive ion mode. And multiple reaction monitoring (MRM) were optimized by flow injection analysis (FIA) mode: source temperature (TEM), nebulizer (NEB), curtain (CUR) and auxiliary (AUX) gas were set at 40, 40, 20 and 40 psi, respectively. Nitrogen gases were used in collision and curtain gases while zero air was used as source gas. Determinations of atorvastatin and orthhydroxy atorvastatin were based on the internal standard method, using chlorzoxazone as the IS. Column oven temperature $(40 \circ \mathrm{C})$ and injection volume $(10 \mu \mathrm{L})$ was maintained throughout the analysis. MRM mode was used to qualify at $\mathrm{m} / \mathrm{z} 559.3 \square \mathrm{m} / \mathrm{z} 575.3 \square \mathrm{m} / \mathrm{z} 170.1$, and quantify the target compounds at $\mathrm{m} / \mathrm{z}$ 440.0, m/z 440.1, m/z 114.3 for atorvastatin, orth-hydroxy atorvastatin and chlorzoxazone, respectively. The main working parameters of the MS were optimized as following: lon spray voltage was at $5500 \mathrm{~V}$ and source temperature was at $450 \circ \mathrm{C}$. Nitrogen gas was used as sheath gas ( 55 arbitrary units) and auxiliary gas (15 arbitrary units). Declustering potential (DP) at 55V, the collision energy was optimized at $26 \mathrm{eV}$ for atorvastatin, $30 \mathrm{eV}$ for orth-hydroxy atorvastatin, and $20 \mathrm{eV}$ for IS, respectively. The developed method and above mentioned instruments were used for all the experiments conducted in this study and the specifications of other instruments used are mentioned at their respective places.

\section{METHOD VALIDATION}

Selectivity. The selectivity was defined as the absence of interference from the blank serum components at the retention times of atorvastatin, orth-hydroxy atorvastatin and IS using the proposed extraction procedure and UPLC/MS conditions. Six different blank serum samples (did not receive the treatment of atorvastatin) from hospitalized volunteers with normal renal function or uremia hemodialysis patients were evaluated to assess the selectivity of the method.

Linearity. Linearity was determined on 3 different days using fresh preparations. The calibration curves were prepared by spiking matrix sample over a concentration range of $0.05-20.00 \mathrm{ng} / \mathrm{mL}$ for atorvastatin and orth-hydroxy atorvastatin. The slopes, intercepts and correlation coefficients $\left(r^{2}\right)$ were obtained by 
weighted $\left(1 / \mathrm{x}^{2}\right)$ linear regression analysis ${ }^{[6]}$. The calibration curve was developed using the following criteria: (1) the mean value should be within $\pm 15 \%$ of the theoretical value, except at LLOQ, where it should not deviate by more than $\pm 20 \%$; (2) the precision around the mean value should not exceed a $15 \%$ coefficient of variation (CV), except for LLOQ, where it should not exceed $20 \%{ }^{[6]}$.

Detection limits (Lower limit of quantification, LLOQ). Detection limits was determined as a signal/noise ratio being at least 5 . Analyte peak should be identifiable, discrete, and reproducible with a precision of $20 \%$ and accuracy of $80-120 \%[7]$.

Matrix effects. Matrix effects were investigated using the post-extraction spike method, which measured the ionization recovery, determined by the ratio of the peak area of analytes spiked after extraction to the peak area of standard solutions at the same concentration ${ }^{[8]}$.

To evaluate the relative matrix effects, calibration curves from six serum batches were constructed, and then the precision (expressed by RSD) values for slopes were calculated. The RSD should not exceed 3$4 \%$ in order to confirm a method to be practically free from relative matrix effect.

Accuracy and Precision. The intra-day and inter-day assay precisions were determined using the CV (\%), and the accuracies were expressed as the percent difference by using the following formula[9]:

\section{$\underline{\text { measured concentrat ion }} \times 100 \%$ \\ nominal concentrat ion}

Intra-day assay precision and accuracy were calculated using 6 determinations of the $3 \mathrm{QC}(0.15,5.00$, $15.00 \mathrm{ng} / \mathrm{mL}$ for atorvastatin and orth-hydroxy atorvastatin) during a single analytical run. Inter-day assay precision and accuracy were calculated by analyzing the $3 \mathrm{QC}(\mathrm{n}=6)$ on 3 separate days. Precision should not exceed $15 \%$ and bias should be within 85 and $115 \%$.

Recovery. The recovery was determined by the analysis of serum samples at 3 concentrations $(0.15,5.00$, $15.00 \mathrm{ng} / \mathrm{mL}$ for atorvastatin and orth-hydroxy atorvastatin). Each concentration level was extracted and analyzed, and the responses compared with those of non-extracted standards corresponding to $100 \%$ recovery.

Stability. Stability procedures were defined to evaluate the stability of the analytes during sample collection and handling ${ }^{[10]}$.

Short-stability was determined by assaying the 3 QC samples at room temperature after thawing 1 hour for the short stability.

Freeze-thaw stability was determined by assaying the 3 QC over 3 freeze-thawing cycles. The QC samples were stored at $-80^{\circ} \mathrm{C}$ for 24 hours and thawed at room temperature. When completely thawed, the samples were refrozen for 24 hours. The freeze-thawing cycles were repeated two times and the analysis 
conducted at the end of the third cycle. The measured concentration was then compared to the theoretical concentration ${ }^{[11]}$.

Post-preparative stability was determined by assaying the $3 \mathrm{QC}$ samples in autosampler at $+4^{\circ} \mathrm{C}$ by injecting extracts immediately after preparation and re-injecting 2 and 12 hours later; Long-term stability was determined from serum samples stored at $-80^{\circ} \mathrm{C}$ for 30 days ${ }^{[12]}$.

\section{DETERMINATION OF DRUG CONCENTRATION IN ULTRAFILTRATE}

The drug-free human plasma of $500 \mu \mathrm{L}$ volume were transferred to Amicon centrifree micropartition devices and centrifuged at $6000 \mathrm{r} \cdot \mathrm{min}-1\left(37^{\circ} \mathrm{C}\right)$ for $15 \mathrm{~min}$, and all of the original volume of plasma was collected as an ultra-filtrate. The atorvastatin standard solutions was diluted with ultra-filtrate to 0.05 , $0.50,2.00 \mathrm{ng} . \mathrm{mL}-1$, respectively. The drug-containing ultra-filtrate was processed and analyzed same as the plasma samples according to the method described before, parallel determination 6 times. The atorvastatin concentration in the ultra-filtrate was calculated using a standard curve of the plasma samples, and compared with the actual concentrations.

\section{PLASMA PROTEIN BINDING STUDY OF ATORVASTATIN IN VITRO}

The percentage of plasma protein binding (PPB) of atorvastatin in vitro was performed by ultrafiltration technique. Excessive centrifugation speed or excessively long time may cause damage to the ultrafiltration membrane and leak the sample, or destroy the binding of drug and plasma protein, and affect the final result. The time and speed of the centrifuge were examined and finally determined 6000 $\mathrm{r} / \mathrm{min}$ and $15 \mathrm{~min}$.

A certain amount of atorvastatin working solutions were added to the drug-free plasma (From volunteers with normal renal function and uremia hemodialysis patients, respectively.), and prepared a drugcontaining plasma with a concentration of $0.05,2.00$, and $10.00 \mathrm{ng} / \mathrm{mL}$, respectively. To achieve equilibrium between the drug and plasma proteins, the spiked drug-contained plasma samples were incubated at $37^{\circ} \mathrm{C}$ for 60 min prior. An aliquot $(500 \mu \mathrm{L})$ of the obtained plasma was transferred to the centrifugal filter unit. Samples of $500 \mu \mathrm{L}$ volume were transferred to Amicon centrifree micropartition devices and centrifuged at $6000 \mathrm{r} \cdot \mathrm{min}-1\left(37^{\circ} \mathrm{C}\right)$ for $15 \mathrm{~min}$, and all of the original volume of plasma was collected as an ultra-filtrate. The ultra-filtrate volume was calculated by weight loss method and the protein binding rate was calculated according to the following formula. Plasma samples without ultrafiltration and their respective ultra-filtrates were analyzed by UPLC-MS/MS method. The percentage of PPB was calculated as follows:

PPB $=\left[\left(C_{\text {ultra-filtrate }}{ }^{*} V_{\text {ultra-filtrate }}\right) / V_{\text {Ultra-filtered plasma }}\right] / C_{\text {plasma }}$

THE PPB AND METABOLISM RATIO STUDY IN VIVO 
Age $>18$, the serum creatinine (SCr) of patients with normal renal function was $54 \sim 106$ and $44 \sim 97$ $\mu \mathrm{mol} / \mathrm{L}$ for male and female, respectively; and the glomerular filtration rate (GFR) of uremia patients $<15$ $\mathrm{mL} /(\mathrm{min} \cdot 1.73 \mathrm{~m} 2)$; excluded emergency, adverse reactions to atorvastatin and organ-transplants patients .All of the patients taking atorvastatin calcium tablets (Lipitor ${ }^{\circledR}$ ) for $\geq 7$ days orally ( $>5 \mathrm{t} 1 / 2$, steady-state plasma concentration), the frequency of administration was once per night (qn.), dose for $20 \mathrm{mg}$, venous blood was collected at 6 am the next day and placed in a heparin anticoagulation tube. Determination of the free and combined concentrations of atorvastatin and its metabolite in samples by UPLC-MS/MS method established by this method, and calculated of the percentage of PPB and metabolite/prototype ratio, respectively. This study was designed in accordance with legal requirements and the declaration of Helsinki and approved by the local research ethics committee.

\section{Results}

\section{METHOD VALIDATION}

Selectivity. The chromatograms of the blank serum samples from drug-free hospitalized volunteers and uremia hemodialysis patients were presented in figure 1. No interferences were observed at the retention times of atorvastatin, orth-hydroxy atorvastatin and IS.

Linearity. The best linear fit and least-squares residuals for the calibration curve were achieved with a $1 / x^{2}$ weighting factor with linear ranges of $0.05-20.00 \mathrm{ng} / \mathrm{mL}$ for atorvastatin and $0.05-20.00 \mathrm{ng} / \mathrm{mL}$ for orth-hydroxy atorvastatin, respectively. The linear regression equations were $y=0.09914 x+0.03348$ $\left(r^{2}=0.9980\right)$ for atorvastatin and $y=0.10033 x+0.01922\left(r^{2}=0.9976\right)$ for orth-hydroxy atorvastatin, respectively. The intercept coefficients were not significant $(p=1.000$ for atorvastatin, $p=0.948$ for orthhydroxy atorvastatin).

Detection limit. The lower limit of quantification was $0.05 \mathrm{ng} / \mathrm{mL}$ for atorvastatin $(\mathrm{S} / \mathrm{N}>5)$ and 0.05 $\mathrm{ng} / \mathrm{mL}$ for orth-hydroxy atorvastatin $(\mathrm{S} / \mathrm{N}>5)$, respectively.

Matrix effects. The $3 \mathrm{QC}$ standard solutions $(0.15,5.00,15.00 \mathrm{ng} / \mathrm{mL}$ for atorvastatin and orth-hydroxy atorvastatin) were added into 3 different blank serum in triplicate, separately. The absolute value and ratio of the peak area of analytes spiked after extraction to those of standard solutions were calculated. The results showed that the matrix effects of atorvastatin and orth-hydroxy atorvastatin on the internal standard-corrected samples were $96.23 \%-104.37 \%$ and $92.64 \%-103.59 \%$, respectively. RSD(\%) were both in the normal range (not exceed 3-4\%). Therefore, the assay established in this experiment was not affected by the matrix effect.

Accuracy, Precision and Recovery. The results of recovery, intra-day and inter-day precision and accuracy of atorvastatin and orth-hydroxy atorvastatin were given in Table 1. 
Table 1

Precision and recoveries for the analysis of atorvastatin, ortho-hydroxy atorvastatin in uremia hemodialysis patients plasma $(n=6)$

\begin{tabular}{|lllllll|}
\hline Item & $\begin{array}{l}\text { Concentration } \\
(\mathbf{n g} / \mathbf{m L})\end{array}$ & $\begin{array}{l}\text { Intra-day } \\
(\mathbf{m})\end{array}$ & $\begin{array}{l}\text { RSD } \\
\mathbf{( \% )}\end{array}$ & $\begin{array}{l}\text { Inter-day } \\
\mathbf{( \pm s})\end{array}$ & $\begin{array}{l}\text { RSD } \\
(\%)\end{array}$ & $\begin{array}{l}\text { Recovery } \\
( \pm \mathbf{s})\end{array}$ \\
\hline Atorvastatin & 0.15 & $0.143 \pm 0.009$ & 3.35 & $0.147 \pm 0.006$ & 4.23 & $84.1 \pm 2.3 \%$ \\
\hline 5.00 & $5.004 \pm 0.043$ & 1.43 & $5.039 \pm 0.047$ & 2.85 & $76.5 \pm 1.1 \%$ \\
\hline $\begin{array}{l}\text { Orth-hydroxy } \\
\text { Atorvastatin }\end{array}$ & 15.00 & $14.88 \pm 0.16$ & 0.65 & $14.85 \pm 0.21$ & 1.66 & $82.0 \pm 1.7 \%$ \\
& 0.15 & $0.143 \pm 0.008$ & 4.56 & $0.146 \pm 0.005$ & 5.22 & $84.4 \pm 2.5 \%$ \\
& 5.00 & $4.915 \pm 0.080$ & 2.08 & $4.963 \pm 0.11$ & 2.34 & $79.1 \pm 1.5 \%$ \\
\hline
\end{tabular}

Stability. Short-term stability and three consecutive freeze-thawing cycles showed no significant degradation for atorvastatin and orth-hydroxy atorvastatin. The result of post-preparative stability showed that atorvastatin and orth-hydroxy atorvastatin were still stable after 2 and 12 hours' operation. The serum samples stored at $-80^{\circ} \mathrm{C}$ were found to be stable for 30 days.

\section{DETERMINATION OF DRUG CONCENTRATION IN ULTRAFILTRATE}

The RSD between concentration calculated from the standard curve (plasma) and the actual concentration was less than $5.57 \%$. The statistics analysis showed that there were no significant difference $(P<0.05)$. This method is suitable for the determination of free atorvastatin concentration in the ultrafiltrate.

\section{PROTEIN BINDING RATE EXPERIMENT}

The study in vitro(Table 2) showed that the plasma protein binding rate in uremia patients plasma was reduced by about $10 \%$ compared with those with the plasma from normal renal function person. The average protein binding rate of plasma in uremia patients plasma was $86.58 \pm 2.04 \%$, RSD $(\%)=1.98$, while the protein binding rate in plasma of patients with normal renal function was $97.62 \pm 1.96 \%$, RSD $(\%)=2.04, T$ test analysis showed that there was no significant difference in the protein binding rate of atorvastatin at different concentrations $(P>0.05)$, but there was a significant difference in the protein binding rate of different types of plasma $(P<0.05)$. 
Table 2

The protein binding rate of atorvastatin in uremia patients and health volunteers plasma of different concentrations $(n=6)$

\begin{tabular}{|c|c|c|c|c|c|c|}
\hline \multirow{2}{*}{$\begin{array}{l}\text { Concentration } \\
\left(\mathrm{ng} \cdot \mathrm{mL}^{-1}\right)\end{array}$} & \multicolumn{3}{|c|}{ Health human plasma } & \multicolumn{3}{|c|}{ Uremia patients plasma } \\
\hline & 1.00 & 5.00 & 10.00 & 1.00 & 5.00 & 10.00 \\
\hline \multirow{6}{*}{$\begin{array}{l}\text { Protein Binding } \\
\text { Rate }\end{array}$} & $98.33 \%$ & $98.81 \%$ & $96.56 \%$ & $88.45 \%$ & $86.33 \%$ & $87.36 \%$ \\
\hline & $99.04 \%$ & $96.56 \%$ & $97.37 \%$ & $87.83 \%$ & $85.84 \%$ & $86.51 \%$ \\
\hline & $96.27 \%$ & $97.77 \%$ & $95.67 \%$ & $84.92 \%$ & $84.49 \%$ & $85.45 \%$ \\
\hline & $97.76 \%$ & $97.83 \%$ & $98.86 \%$ & $86.43 \%$ & $89.33 \%$ & $87.84 \%$ \\
\hline & $95.44 \%$ & $98.58 \%$ & $99.05 \%$ & $89.56 \%$ & $84.56 \%$ & $88.65 \%$ \\
\hline & $97.86 \%$ & $98.68 \%$ & $96.73 \%$ & $84.56 \%$ & $83.78 \%$ & $86.47 \%$ \\
\hline Mean & $97.62 \%$ & & & $86.58 \%$ & & \\
\hline $\mathrm{RSD}(\%)$ & 1.96 & & & 2.04 & & \\
\hline
\end{tabular}

A total of 45 uremia hemodialysis patients were included with a median age of 71 years old (range: 6085 ) with a dose of $20 \mathrm{mg} /$ day, and 35 patients with normal kidney function were included with a median age of 67 years old (range: 50-80) with a dose of $20 \mathrm{mg} /$ day. Our studys showed that the mean total plasma concentration of atorvastatin in the normal renal function group is $(7.45 \pm 4.68) \mathrm{ng} \cdot \mathrm{mL}-1$, and $(5.97 \pm 4.20) \mathrm{ng} \cdot \mathrm{mL}-1$ for uremia patients group(Figure 2). But T-test analysis showed that there is no significant difference between the two groups $(P=0.239>0.05)$, which mean uremia had no significant effect on the total plasma concentration atorvastatin. But there was a significant difference in the free concentration of atorvastatin for the two groups $(P=0.013<0.05)$, which was $(0.38 \pm 0.38)$ and $(0.78 \pm$ 0.94) $\mathrm{ng} \cdot \mathrm{mL}-1$ for the normal renal function group and the uremia patients group, respectively (Figure 3 ). The free concentration was significantly increased due to the decrease in protein binding rate. The mean PPB rate of atorvastatin in the normal renal function group was $95.33 \pm 2.36 \%$, which proved that the method established in our study was trustworthy. And the mean PPB rate of atorvastatin in the uremia patients group was only $85.06 \pm 8.32 \%$ (Figure 4). The plasma protein of atorvastatin in uremia patients group was significantly lower than the renal function group $(P=0.00<0.05)$. The results were basically consistent with experiments in vitro.

Owing to the renal filtration function loss and negative nitrogen balance in uremic patients, the physiological state is often monitored by albumin and creatinine values. The serum creatinine value reflects the level of toxin accumulation in uremic patients, while albumin concentration may affect the protein binding rate of the drug. 
The biochemical indicators of subjects enrolled in this study were shown in the Table 3 and Figure 5. Logistic regression analysis showed that the most significant factor related to the protein binding rate of atorvastatin was creatinine level $(P<0.01)$, and the protein binding rate decreases with increasing creatinine until it is stable at nearly $80 \%$. The correlation of the atorvastatin's protein binding rate and plasma albumin level was not significant in our study $(P>0.05)$.

Table 3

The biochemical indicators of uremia patients and health volunteers

\begin{tabular}{|llll|}
\hline Categories & Mean & P \\
\cline { 2 - 3 } & Healthy volunteers & Uremic patients & \\
\hline AST & $15.77 \pm 3.21$ & $16.02 \pm 4.65$ & $>0.05$ \\
\hline ALT & $14.55 \pm 2.88$ & $15.89 \pm 3.93$ & $>0.05$ \\
\hline albumin(g/L) & $38.99 \pm 6.43$ & $35.37 \pm 5.78$ & $<0.05$ \\
\hline creatinine( $\mu \mathrm{mol} / \mathrm{L})$ & $61.65 \pm 19.82$ & $385.89 \pm 223.77$ & $<0.01$ \\
\hline
\end{tabular}

Figure 6 showed the distribution of trough concentrations of atorvastatin and orth-hydroxy atorvastatin.. The average plasma concentration of orth-hydroxy atorvastatin in the patients with normal kidney function was $(7.75 \pm 4.93) \mathrm{ng} / \mathrm{mL}$, and in the uremia hemodialysis patients was $(4.97 \pm 4.89) \mathrm{ng} / \mathrm{mL}$, T-test analysis results showed that there was significant difference between the two groups of plasma concentrations $(P<0.05)$. The mean metabolite/prototype ratio of atorvastatin for patients with normal renal function and uremia were 1.085 and 0.974 , respectively, and there was a significant difference $(P<0.05)$. The metabolite/prototype ratio of the uremia was lower than the normal renal function group, suggesting that the metabolic process of atorvastatin in uremic patients may be inhibited.

\section{Discuss}

The binding of the drug and plasma protein in the human body is in a dynamic equilibrium, and the protein binding of the drug is in a constant state when the physiological condition is stable. The concentration of free drugs is a direct basis for drug safety and pharmacodynamic evaluation. However, in some special diseases, changes in physiological conditions will affect the protein binding rate, at this time the free concentration will change significantly. At this point, although the total concentration of the drug is still within the effective therapeutic concentration range, the patient shows obvious individual differences in pharmacodynamics, and the pharmacological effect or degree of toxic side effects changes significantly ${ }^{[13]}$. Therefore, further study on the differences and regularity of drug binding and free drug concentration in special populations is of great significance for ensuring the safety and effectiveness of clinical drugs.

Chronic kidney disease is a common clinical chronic disease that eventually develops into end-stage renal disease known as uremia. When uremia happened, the complications such as gastrointestinal dysfunction, autonomic neuropathy, liver dysfunction, changes in the internal environment, affecting drug 
absorption, protein binding, the expression of drug transporter, metabolic enzyme activity and may hinder drug metabolism and excretion, ultimately affecting drugs metabolic dynamics behavior. The uremic toxins such as aromatic amino acids, peptides or metabolites accumulate in the body, and bind to albumin in serum to form most of the large and medium molecules that are almost impossible to remove by blood purification methods ${ }^{[14]}$. The accumulation of uremic toxins in uremic patients, changes in the binding site for albumin, or the conformation of the binding protein, or the deterioration of albumin, leading to a decrease in protein affinity, may influence the protein binding rate of certain drugs ${ }^{[15,16]}$. In addition, changes in body fluid $\mathrm{pH}$, hypoproteinemia during uremia also influenced the protein binding rate of drugs ${ }^{[16]}$. In our study, there was no significant effect of the total plasma concentration of atorvastatin between patients with normal kidney function and uremia hemodialysis patients, but the protein binding rate of atorvastatin in uremia plasma is significantly reduced, and the free drug concentration is increased by nearly two-fold, which may increase the distribution concentration of the drug in the tissue, thereby increasing the risk of adverse reactions.

But atorvastatin's metabolic processes in uremia hemodialysis patients were inhibited. Atorvastatin is mainly metabolized by the CYP3A isoenzyme in the liver, and can be absorbed by P-gp secretion and H+MCT co-transporter on the Caco-2 cell lumen side, or as a substrate for OATP1B1, OATP1A4 and OATP1B2 [17]. Studies had shown that uremic toxins affect the mRNA, protein expression and function of metabolic enzymes and drug transporters. Reduced non-renal clearance of drugs in the body, manifested by increased bioavailability and/or elevated plasma concentrations $[18,19]$. Changes in the activity of renal transporters affect the pharmacokinetics of drugs that are primarily eliminated from the kidneys, and changes in liver and intestinal transporter activity affect the pharmacokinetics of non-renal elimination drugs. In addition to changes in liver metabolic enzyme activity, decreased liver drug intake or increased efflux may down-regulate drug metabolism, while drugs absorbed in the intestine need to be metabolized by the gastrointestinal tract before liver metabolism, and the expression of intestinal uptake transporter is up-regulated. Enhanced or reduced efflux transporter activity may increase the bioavailability of certain drugs in uremic patients.

Studies had shown that renal insufficiency affects the activity of CYP450 enzymes ${ }^{[20-26]}$. The total amount of CYP enzyme in mice with renal insufficiency decreased by $47 \%$ and was negatively correlated with renal clearance; the protein expression of CYP2C11, 3A1 and 3A2 was down-regulated by $40 \%, 74 \%$ and $65 \%$, respectively; the mRNA expression levels of CYP1A2, 2C11, 2C29, 3A1, 3A2 and 3A11 were significantly down-regulated ${ }^{[21-23]}$. The activity and protein expression levels of CYP3A and CYP2C11 in the liver of uremic patients decreased with the down-regulation of mRNA expression levels ${ }^{[25]}$. THOMSON [26] found that the blood concentration of CYP3A4 probe drug midazolam in hemodialysis patients increased nearly 6 times compared with normal people, suggesting that liver CYP3A4 enzyme activity is inhibited.

The liver transporter in uremic patients reduces drug intake and increases efflux, which may be one of the causes of drug metabolism down-regulation; the expression and activity of uptake transporters in the 
intestinal tract are up-regulated, and the efflux transporter activity is down-regulated, affecting its transport substrate metabolism and excretion, ultimately affect the drug concentration. Nolin ${ }^{[27]}$ found that the pharmacokinetic parameters of oral administration of midazolam (CYP3A substrate) after hemodialysis in uremic patients were not significantly different from those in healthy subjects, whereas the clearance rate of oral fexofenadine (a common substrate for CYP3A and OATP, P-gp) was reduced by $63 \%$, and fexofenadine did not change significantly in non-hemodialysis uremic patients, while the concentration of fexofenadine in uremic patients treated with hemodialysis and peritoneal dialysis ware significantly increased ${ }^{[28]}$ suggesting that the intestinal and liver transporters were inhibited in uremia. Furthermore, the activity of P-gp and MRP2 in the intestinal tract of rats with renal insufficiency was reduced by $30 \%$ and $25 \%$, respectively, and the protein expression of P-gp, MRP2 and MRP3 were decreased by more than 40\%; the protein expression and mRNA level of the efflux transporter P-gp in the liver of renal dysfunction mice increased significantly, while the uptake transporter OATP2 protein expression decreased by $35 \%$ but the mRNA level did not change significantly ${ }^{[29-31]}$; the expression levels of mRNA and protein in OAT1, OAT2, OAT3, OATP1 and OATP4c1 were down-regulated in the kidney, while the mRNA and protein expression levels of MRP2, MRP3, MRP4 and OATP2 and OATP3 were upregulated ${ }^{[32]}$. Clinical studies have further confirmed this view. Sakurai ${ }^{[33]}$ found that OAT1 mRNA expression was down-regulated in live kidney sections of patients with various renal dysfunctions with varying degrees of renal insufficiency, OAT3 was slightly up-regulated, and there was no significant difference in OAT2 and OAT4 expression, whereas the clearance rate of cefazolin (the anionic drug) was significantly correlated with the expression level of OAT3 mRNA.

In theory, the protein binding rate of atorvastatin is reduced in uremic patients, the concentration of free drug is increased, the amount of drug involved in metabolism is increased, and the metabolic rate should be accelerated, but the metabolism of atorvastatin in uremic patients is inhibited. The concentration of metabolites is also significantly reduced. The presumed reasons may be as follows: 1 . The CYP3A4 enzyme activity in uremic patients was inhibited, and the decrease in activity lead to a decrease in the metabolism of atorvastatin; 2. Atovastatin was mainly transported to the liver through OATP1B3 for metabolism, the activity of OATP transporter in the liver of uremic patients was inhibited, resulting in a decrease in atorvastatin transported to liver cells via OATP, and a decrease in the amounts of drugs involved in metabolism. 3. The degree of inhibition of metabolic enzymes and transporters is greater than the effect of elevated concentrations of free drugs in uremic patients, the final manifestation is that the metabolic process of atorvastatin is inhibited.

In this study, the total concentration of atorvastatin in uremic patients did not change significantly, but due to the decrease of protein binding rate, the concentration of free drug may fluctuate drastically, and the increase of free drug concentration will increase the drug distribution in the liver or muscle tissue, studies had shown that the increased concentration of atorvastatin increases the risk of adverse reactions, such as rhabdomyolysis, liver damage ${ }^{[34]}$. Uremic patients who were taking atorvastatin should be monitored regularly, If there is an unexplained muscle pain, weakness or other adverse reactions, stop 
the drug immediately and report it to the doctor promptly, ensure the safety of medication for this particular group.

\section{Declarations}

\section{Acknowledgements}

First and foremost, I would like to show my deepest gratitude to my supervisor, Mrs. HUANG, a respectable, responsible and resourceful scholar, who has provided me with valuable guidance in every stage of the writing of this thesis. Without her enlightening instruction, impressive kindness and patience, I could not have completed my thesis. Her keen and vigorous academic observation enlightens me not only in this thesis but also in my future study. I would also like to thank all my teachers who have helped me to develop the fundamental and essential academic competence. My sincere appreciation also goes to the teachers and students from the Shandong Provincial Qianfoshan Hospital, who participated this study with great cooperation. Last but not least, I' d like to thank all my friends, for their encouragement and support.

\section{Ethics approval statement}

The experimental protocol was established, according to the ethical guidelines of the Helsinki Declaration and was approved by the Human Ethics Committee of the Shandong Provincial Qianfoshan Hospital. Written informed consent was obtained from individual or guardian participants.

\section{Competing interests}

The authors declare no competing non-financial/financial interests.

\section{Funding}

This work is supported by Science and Technology Development Plan of Shandong Province (2014GGB14721).

\section{Contributions}

$M C$ and WZ contributed to the conception of the study; $H X$ and $M X$ contributed significantly to manuscript preparation; $\mathrm{LW}$ and $\mathrm{BH}$ helped perform the analysis with constructive discussions; $\mathrm{HY}$ and $\mathrm{YZ}$ organized the tables and pictures of the full text. All authors contributed significantly, read and approved the final manuscript.

\section{Availability of data and materials}

All data generated or analysed during this study are included in this published article.

\section{Consent for publication "}


Not applicable.

\section{References}

[1]U.S. Department of Health and Human Services, Food and Drug Administration, Center for Drug Evaluation and Research (CDER), Center for Veterinary Medicine (CVM). Guidance for Industry Bioanalytical Method Validation. 2001.

[2]European Medicines Agency, Committee for Medicinal Products for Human Use. Guideline on bioanalytical method validation. 2012.

[3]Qiu, Sheng, Zhuo, et al. Effects of atorvastatin on chronic subduralhematoma[J]. Medicine, 2017, 96(26):e7290

[4]ARONOV D M. Statins: therapeutic cascade of their effects [J]. Kardiolo-giia, 2004, 44 (10): 85-94.

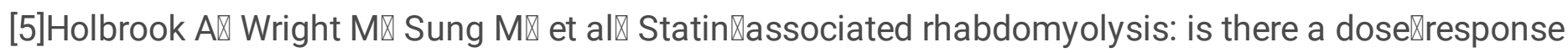
relationship [ J ]. Can J Cardiol区2011区27( 2) : 146 -151凹

[6] Jin H E , Kang I H, Chang K S . Fluorescence Detection of Zabofloxacin, a Novel Fluoroquinolone Antibiotic, in Plasma, Bile, and Urine by HPLC: The First Oral and Intravenous Applications in a Pharmacokinetic Study in Rats[J]. Journal of pharmacy \& pharmaceutical sciences : a publication of the Canadian Society for Pharmaceutical Sciences, SociÃ@tÃ@ canadienne des sciences pharmaceutiques, 2011, 14(3):291.

[7] Al-Akkam E J . Applying of a modified and validated high-performance liquid chromatographic/ultraviolet method for quantification of cetirizine in human plasma for pharmacokinetics studies[J]. Drug Invention Today, 2020, 14(1):45-55.

[8] Primel E G , Fagundes C , Bolzan C M , et al. Evaluation of a modified QuEChERS extraction of multiple classes of pesticides from a rice paddy soil by LC-APCI-MS/MS.[J]. Journal of Agricultural and Food Chemistry, 2011, 59(22):11918-11926.

[9] Yilmaz B , Akba V. Determination and Pharmacokinetics of Ibuprofen in Rabbit Plasma by GC-MS Method[J]. Research Journal of Pharmacy and Technology, 2011, 4(1):52-56.

[10] Shaw L H , Chen W M , Tsai T H . Identification of Multiple Ingredients for a Traditional Chinese Medicine Preparation (Bu-yang-huan-wu-tang) by Liquid Chromatography Coupled with Tandem Mass Spectrometry[J]. Molecules, 2013, 18(9):11281-11298.

[11] Schwilke E W , Karschner E L , Lowe R H , et al. Intra- and intersubject whole blood/plasma cannabinoid ratios determined by 2-dimensional, electron impact GC-MS with cryofocusing.[J]. Clinical Chemistry, 2009(6):1188-1195. 
[12] Pires C C , Kaiser M , Grünspan, Lauren D, et al. Development and validation of a sensitive and selective LC-MS/MS method for the determination of an antimalarial drug candidate in rat plasma, and its application to a preclinical pharmacokinetic study[J]. Acta Chromatographica, 2016, 28(4):1-15.

[13]Piafsky K M. Disease-induced changes in the plasma binding of basic drugs[J]. Clinical Pharmacokinetics, 1980, 5(3): 246-262.

[14]Vanholder R, Baurmeister U, Brunet P, et al. A Bench to Bedside View of Uremic Toxins[J]. Journal of the American Society of Nephrology, 2008, 19(5):863-870.

[15]Thornalley P J, Rabbani N. PROGRESS IN UREMIC TOXIN RESEARCH: Highlights and Hotspots of Protein Glycation in End-Stage Renal Disease[C]// Seminars in Dialysis. PubMed, 2009:400-4.

[16]Sun H, Frassetto L, and Benet LZ. Effects of renal failure on drug transport and metabolism [J]. Pharmacol Ther, 2006, 109: 1-11.

[17]Mladenovska K, Grapci A D, Vavlukis M, et al. Influence of SLC01B1 polymorphisms on atorvastatin efficacy and safety in Macedonian subjects[J]. Die Pharmazie - An International Journal of Pharmaceutical Sciences, 2017.

[18]Vanholder R, Baurmeister U, Brunet P, et al. A Bench to Bedside View of Uremic Toxins[J]. Journal of the American Society of Nephrology, 2008, 19(5):863-870.

[19]Hong Sun, Yong Huang, Lynda Frassetto, et al. Effects of uremic toxins on hepatic uptake and metabolism of Erythromycin[J].Drug Metab Dispos, 2004 , 32(11): 1239-46.

[20]Nolin TD $₫$ Naud J『Leblond FA囚et al『 Emerging evidence of the impact of kidney disease on drug

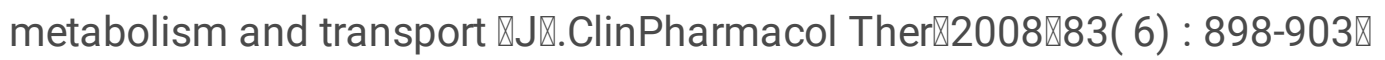

[21]Leblond F A, Giroux L, Villeneuve J P, et al. Decreased in Vivo Metabolism of Drugs in Chronic Renal Failure[J]. Drug Metabolism \& Disposition the Biological Fate of Chemicals, 2000, 28(28):1317-1320.

[22]Leblond F A, Petrucci M, Dubé $P$, et al. Downregulation of intestinal cytochrome p450 in chronic renal failure.[J]. Journal of the American Society of Nephrology, 2002, 13(6):1579-85.

[23]Dani M, Boisvert C, Michaud J, et al. Down-regulation of liver drug-metabolizing enzymes in a murine model of chronic renal failure.[J]. Drug Metabolism \& Disposition the Biological Fate of Chemicals, 2010, 38(3):357-360.

[24]DE MARTIN S,ORLANDO R,BERTOLI M,et al. Differential effect of chronic renal failure on the pharmacokinetics of lidocaine inpatients receiving and not receiving hemodialysis [J]. Clin Phar-macol Ther,2006,80(6) : 597 - 606 . 
[25]VELENOSI T J,FU A Y,LUO S,et al. Down-regulation of hepatic CYP3A and CYP2C mediated metabolism in rats with moderate chronic kidney disease [J]. Drug Metab Dispos,2012,40 (8): 1508 1514.

[26]THOMSON B K,NOLIN T D,VELENOSI T J, et al. Effect of CKD and dialysis modality on exposure to drugs cleared by nonrenal mechanisms [J]. Am J Kidney Dis,2015,65( 4) : 574 - 582.

[27]Nolin T D, Frye R F, Le P, et al. ESRD impairs nonrenal clearance of fexofenadine but not midazolam. [J]. Journal of the American Society of Nephrology Jasn, 2009, 20(10):2269-2276.

[28]Guo X, Meng Q, Liu Q, et al. JBP485 improves gentamicin-induced acute renal failure by regulating the expression and function of Oat1 and Oat3 in rats [J]. Toxicol Appl Pharmacol, 2013, 271: 285-295.

[29]Yu C, Ritter J K, Krieg R J, et al. Effect of chronic renal insufficiency on hepatic and renal udpglucuronyltransferases in rats.[J]. Drug Metabolism \& Disposition the Biological Fate of Chemicals, 2006, 34(4):621-627.

[30]Naud J, Nolin TD, Leblond FA, et al. Current understanding of drug disposition in kidney disease [J]. J Clin Pharmacol, 2012, 52: 10S-22S.

[31]Naud J, Michaud J, Leblond F A, et al. Effects of chronic renal failure on liver drug transporters.[J]. Drug Metabolism \& Disposition the Biological Fate of Chemicals, 2008, 36(1):124.

[32] Naud J, Michaud J, Beauchemin S, et al. Effects of chronic renal failure on kidney drug transporters and cytochrome P450 in rats[J]. Drug Metab Dispos, 2011; 39: 1363-1369.

[33]Sakurai $Y$, Motohashi $\mathrm{H}$, Ueo $\mathrm{H}$, et al. Expression levels of renal organic anion transporters (OAT3) and their correlation with anionic drug excretion in patients with renal diseases[J]. Pharm Res, 2004; 21: 6167.

[34]Schech S, Graham D, Staffa J, et al. Risk factors for statin associated rhabdomyolysis [ J ]. Pharmacoepidemiol Drug Saf 2007, 16: 352 - 358》

\section{Figures}



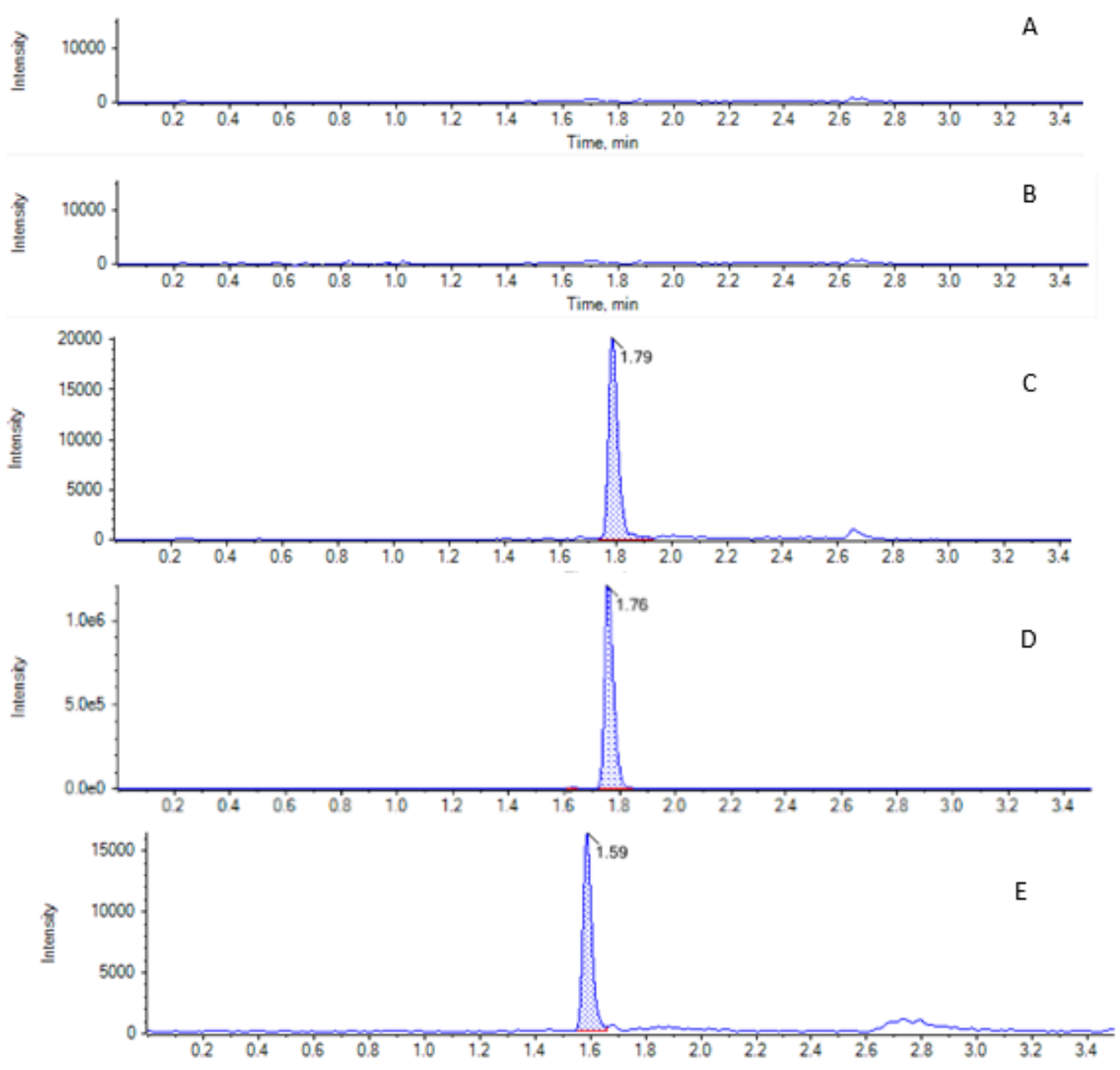

Figure 1

Typical multiple reaction monitoring chromatograms of atorvastatin and internal standard (IS) in human plasma A: Blank plasma samples of volunteers with normal kidney function; B: Blank plasma samples of uremia hemodialysis patients; C: Blank uremia hemodialysis patients plasma spiked with atorvastatin; D: Blank uremia patients plasma spiked with IS 


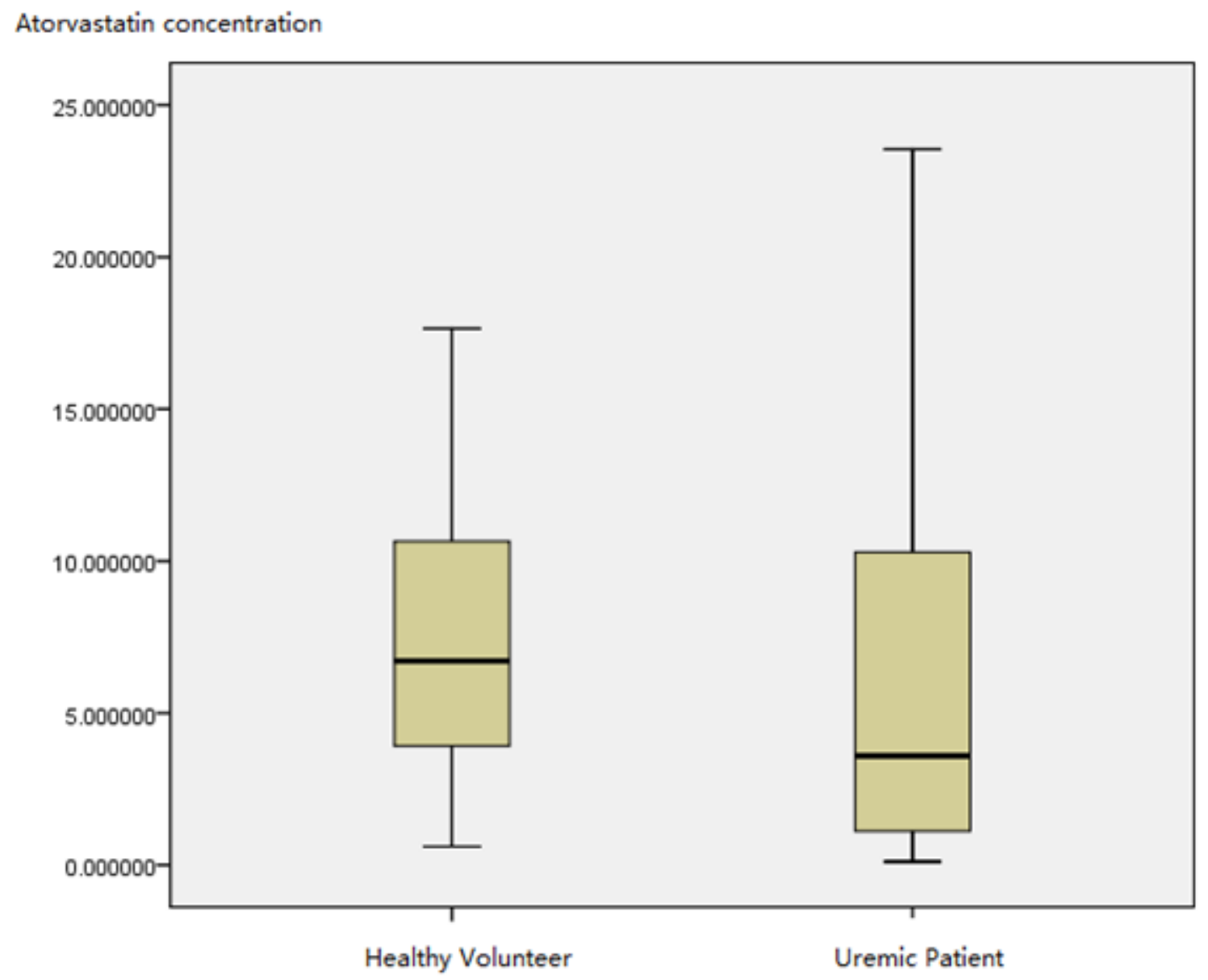

Figure 2

Box-whisker Plot of total plasma concentration of atorvastatin 
Free concentration of atorvastatin

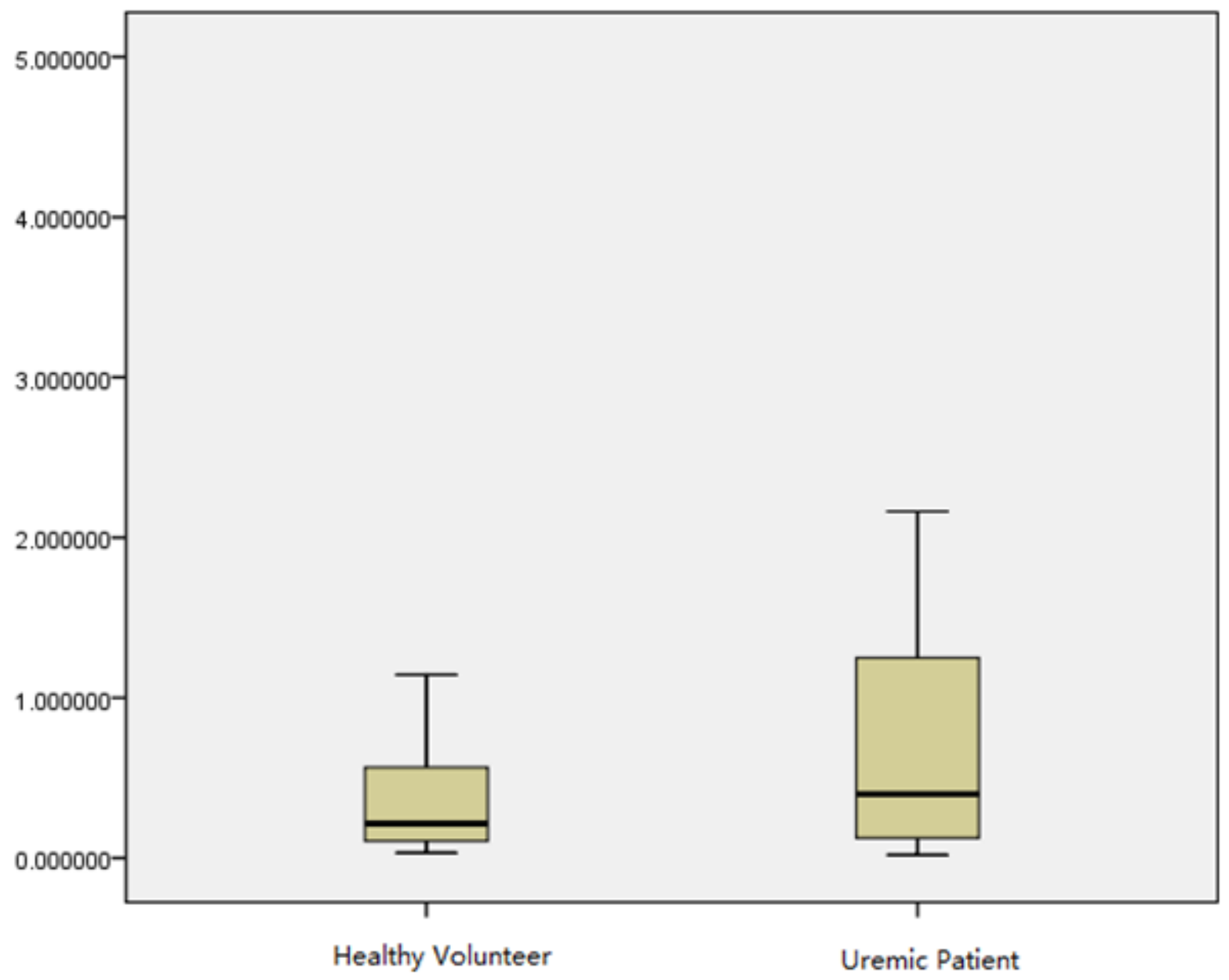

Figure 3

Box-whiskerPlot of free atorvastatin concentration 
Protein Binding Rate of Atorvastatin

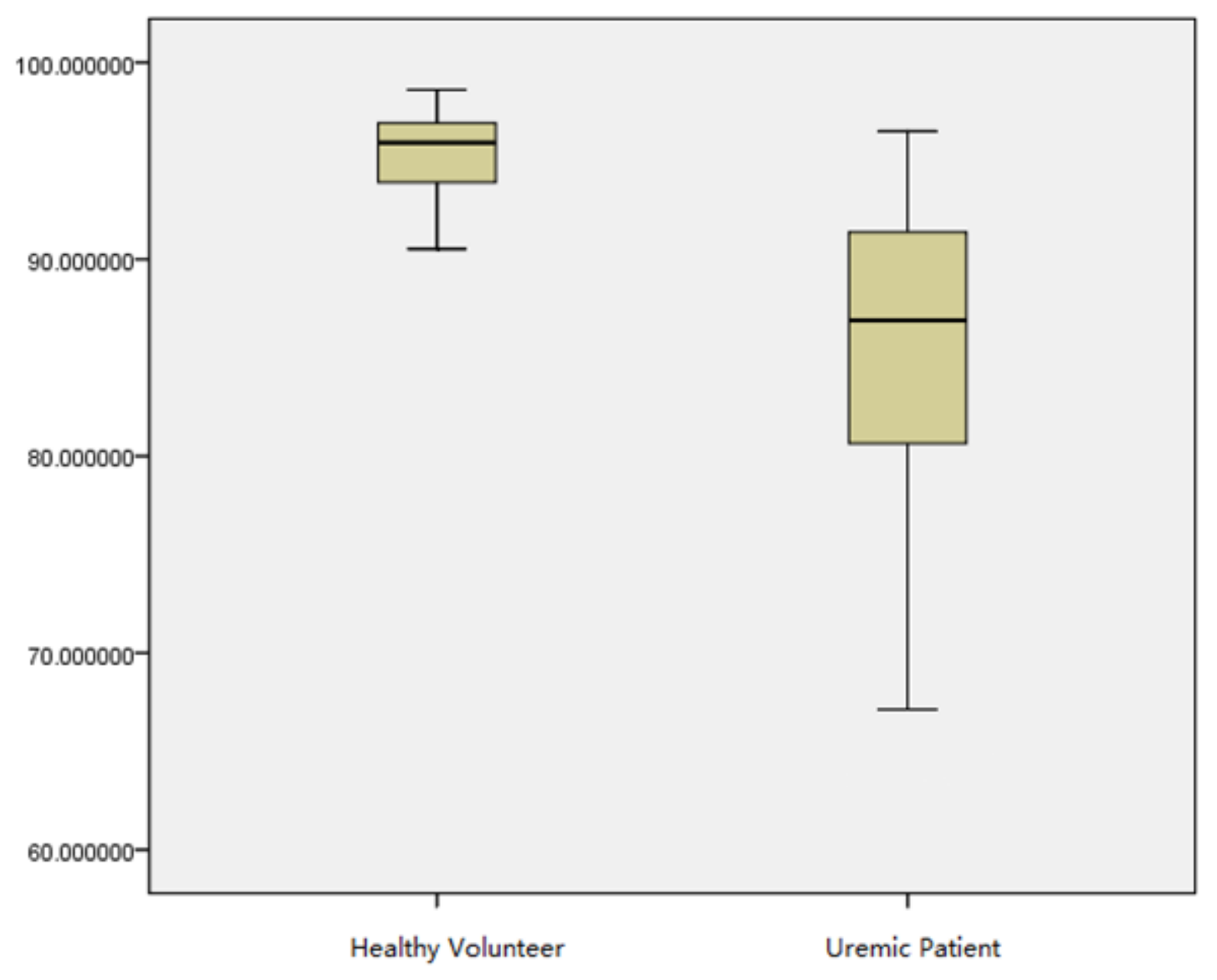

Figure 4

Box-whiskerPlot of protein binding rate of atorvastatin 

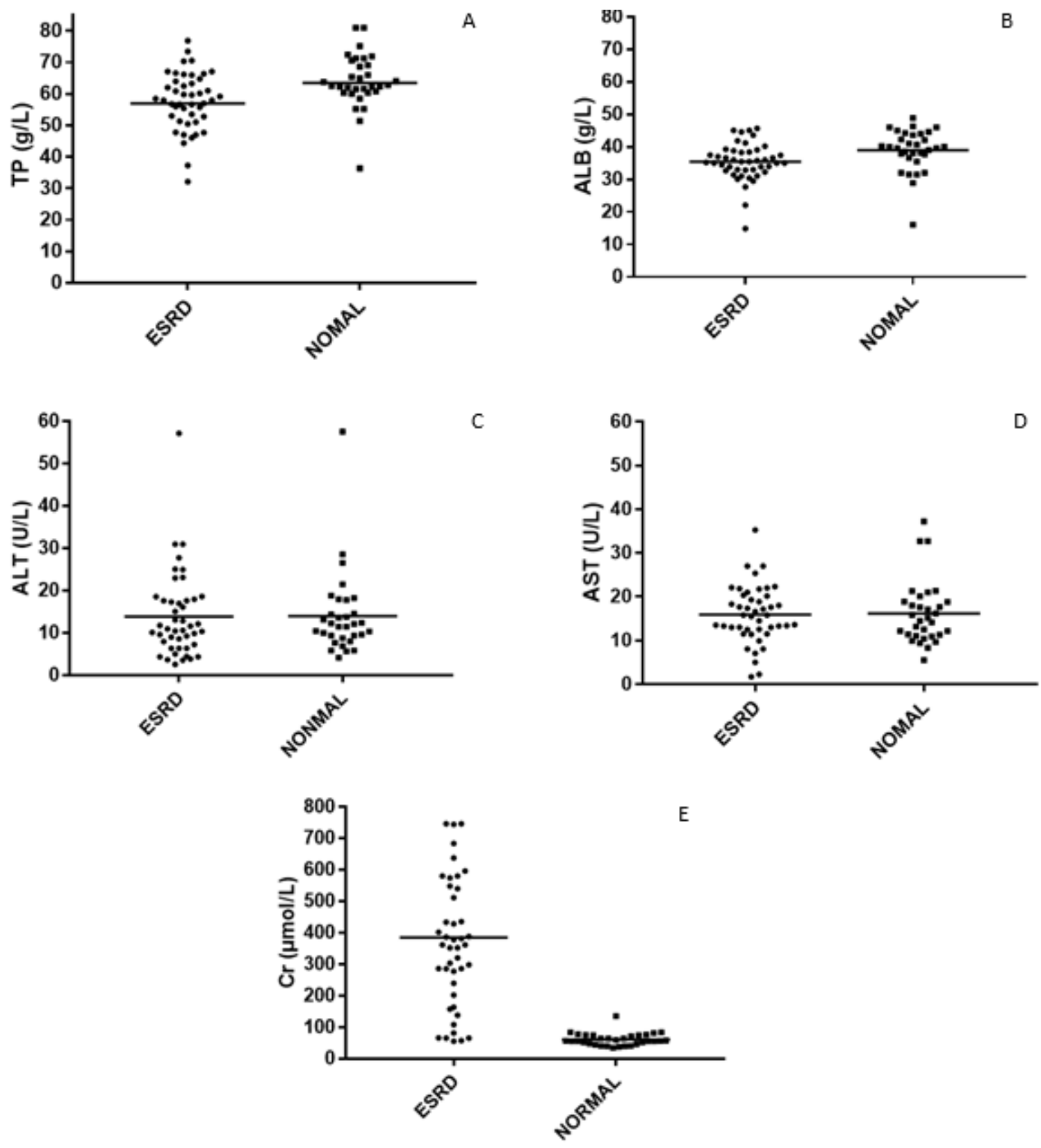

Figure 5

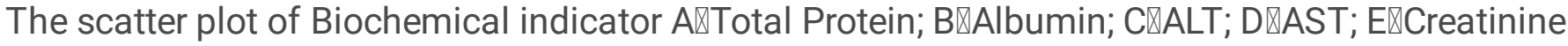




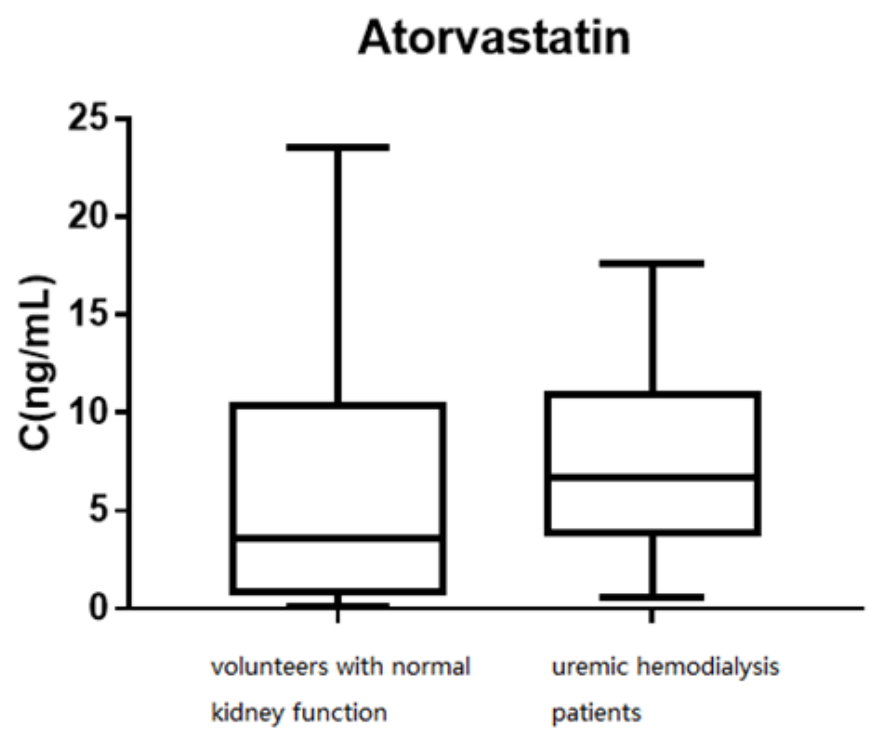

\section{Orth-hydroxy Atorvastatin}

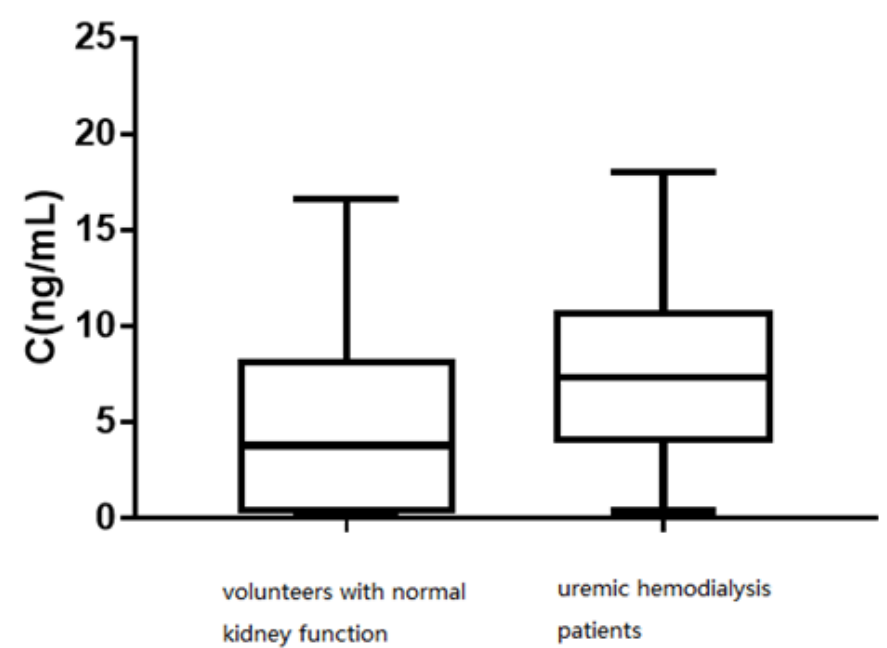

Figure 6

The distribution of trough concentrations for atorvastatin and ortho-hydroxyatorvastatin 